\title{
Tangence
}

\section{Du bon usage des écrivains qui viennent de loin}

\section{Maximilien Laroche}

Numéro 59, janvier 1999

Écrivains d'ailleurs

URI : https://id.erudit.org/iderudit/025988ar

DOI : https://doi.org/10.7202/025988ar

Aller au sommaire du numéro

Éditeur(s)

Tangence

ISSN

0226-9554 (imprimé)

1710-0305 (numérique)

Découvrir la revue

Citer cet article

Laroche, M. (1999). Du bon usage des écrivains qui viennent de loin. Tangence, (59), 20-25. https://doi.org/10.7202/025988ar d'utilisation que vous pouvez consulter en ligne.

https://apropos.erudit.org/fr/usagers/politique-dutilisation/ 


\section{Du bon usage des écrivains qui viennent de loin}

\section{Maximilien Laroche}

On pourra peut-être regretter l'imprécision de cette dénomination: "écrivain qui vient de loin". Mais il sera toujours plus significatif de signaler la provenance lointaine des artistes que l'éloignement de l'objectif qu'on semble leur fixer: l'identification au lieu d'habitation. Et puis double désavantage de l'épithète "néoquébécois", qui n'est qu'un calque de "néo-canadien" et une façon de pratiquer l'idéologie de rattrapage en matière d'immigration, c'est qu'elle pourrait faire redouter aux uns d'être toujours nouveaux, sans cesse inachevés, alors que les autres devraient craindre de toujours passer pour anciens, voire arriérés. PhilippeAubert de Gaspé renvoyait déjà à cette opposition entre Nouveaux et Anciens Canadiens. On n'en aurait donc pas encore fini avec ce dilemme. Mieux vaut considérer les choses autrement.

\section{Éxilés et immigrants}

L'immigrant est coupé d'un espace géographique et surtout démographique. L'éxilé, lui, est en rupture avec son espace subjectif et avec un temps historique car l'incertitude qu'il ressent porte davantage sur le temps que sur l'espace. Il ne sait pas quand ni s'il pourra rentrer chez lui, c'est-à-dire dans cet espace intérieur qui le loge dans un monde où il se reconnaît. Une chanson célèbre le fait bien comprendre:

Un Canadien errant, Banni de ses foyers, Parcourait en pleurant

Des pays étrangers.

Un jour, triste et pensif, Assis au bord des flots, Au courant fugitif

Il adressa ces mots:

Si tu vois mon pays,

Mon pays bienheureux,

Vas, dis à mes amis

Que je me souviens d'eux... 
L'immigrant s'inquiète plutôt d'acquérir la nouvelle identité qui l'insérera dans un nouveau monde. Ainsi on a tort de confondre exilé et immigrant puisqu'on ne le fait, pour parler méthodologie, qu'en traitant des questions d'esthétique avec des lunettes de juriste, en classant des écrivains dans des catégories du service d'immigration.

Celui qui s'exile change de psychologie et devient l'éloigné, le séparé. Cela, en principe, n'a rien à voir avec la condition juridique de l'immigrant qui est celui qui effectue un échange. Il quitte un pays pour un autre, une terre natale pour celle d'une renaissance, une nationalité pour une citoyenneté. On peut être exilé sans immigrer. Émile Nelligan n'a sans doute pas encore trouvé plus éxilé que lui dans un Québec qu'il n'a pourtant jamais quitté. L'immigrant peut fort bien ne pas être un exilé. Parlez-en à ces immigrants-investisseurs, surtout ceux de HongKong, qui font la navette entre leur pays d'origine et celui de leur nouvelle citoyenneté, à la grande satisfaction d'ailleurs des gouvernements des pays entre lesquels ils oscillent.

Émile Nelligan, au Québec, Magloire St-Aude, en Haiti, ont été tous deux des exilés de l'intérieur. En France, Rimbaud a d'abord été un exilé qui est allé jusqu'à dire: "Je est un autre." Puis il a émigré en Abyssinie et à ce moment-là il n'a plus été qu'un trafiquant d'armes.

On se trompe donc, dans le cas des artistes, et en particulier des écrivains, en confondant en un seul et même vocable celui de néo-québécois -, deux statuts distincts. Celui qui s'exile subit une perte pour laquelle il ne peut rechercher et obtenir qu'une compensation. Celui qui émigre effectue un troc dont il est en droit d'attendre un bénéfice. Entre les deux types de passages, il y a une différence d'échelles dans les équivalences. Une compensation n'établit d'équivalence que par médiation, transposition, par une correspondance de type analogique, subjectif. Un troc, un échange, s'effectue sur une base conventionnelle, selon un contrat de nature juridique et formelle qui garantit les bénéfices escomptés.

Là où pour l'exilé il doit être question de transculturation, pour l'émigré on parle d'adaptation ou d'intégration. Et dans ce dernier cas, les cadres de l'exercice sont mieux définis. Il y a diverses chartes de droits et tout un arsenal de règles de sécurité sociale, pour ne pas parler simplement des dispositions de police 
et d'immigration, qui balisent le parcours de l'immigrant. Rien de comparable pour l'exilé dont la trajectoire est imprévisible.

Paradoxalement, c'est dans le cas de l'immigrant qu'il est le plus difficile de bien faire une comptabilité, c'est-à-dire d'envisager clairement des profits à tirer. Comment évaluer, pour un pays, le bénéfice que constituent les immigrants qu'il accueille? Va-t-on pouvoir estimer l'apport de ceux qui nous ont fait connaître la pizza, le chow mein ou le riz basmati? Au Brésil, on reconnaît que l'efficacité des jardiniers japonais a nettement amélioré, depuis leur arrivée, la production des fruits et légumes. Mais estce là leur unique apport ou même le plus significatif? Un récent reportage de la chaîne anglaise de Radio-Canada annonçait qu'en Grande-Bretagne les plats au cari étaient en train de détrôner les bons vieux roast-beef. Et un commentateur d'origine indienne supputait comme conséquence de cette nouvelle mode culinaire que l'estomac ne pouvait pas changer sans que le cerveau, lui aussi, ne connaisse quelques transformations.

Les coûts et bénéfices de l'immigration, pour être plus identifiables, ne sont finalement évaluables qu'en termes de transculturation, c'est-à dire dans la perspective qui fait toujours de la présence de l'étranger un facteur de changement pour tout le monde.

\section{Comparants et comparés}

L'anthropologue cubain Fernando Ortiz a proposé de remplacer le mot "acculturation" par celui de "transculturation" afin de nous rendre plus sensibles aux échanges réciproques, et donc aux pertes et aux gains occasionnés par la cohabitation de deux cultures dans chacune d'elles mais aussi à la transformation opérée en chacune d'elles et finalement à leur fusion en une nouvelle culture, certes tributaire des deux premières, mais offrant aussi des synthèses inédites. Et c'est à cela que l'on devient sensible quand il s'agit de l'exilé, de l'artiste qui vient de loin.

Un écrivain venu de loin est forcément un "compareur" qui nous incite à en devenir un nous aussi puisqu'il fait le pont entre ici et là-bas, entre autrefois et aujourd'hui. Les personnages exotiques qu'il nous présente nous forcent à les rapporter à ceux qui nous sont familiers, à l'image que nous projetons dans les figures de nos désirs que nous voyons désormais mis en parallèle avec d'autres désirs, d'autres images. 
Nous ne pouvons, à lire cet écrivain, que l'imiter, sans forcément le suivre. Peut-être pour mieux le contredire, sûrement pour tester ses évaluations et aussi les nôtres. Mais alors même que nous le suivrions, nous laissant aller au fil de sa nostalgie, a beau mentir qui vient de loin! nous ne ferions que suivre la pente de nos plus secrets désirs. Et ainsi nous nous révélerions à nousmêmes des penchants inconnus, ignorés, cachés ou refoulés.

Si n'importe quelle ouvre est un miroir pour le lecteur, celle qui apporte un parfum de loin, la couleur d'un ailleurs l'est doublement. Je ne sais plus où en est la querelle sur le statut de Louis Hémon, écrivain québécois ou non, mais sa Maria Chapdelaine a servi de révélateur aux lecteurs du Québec et la meilleure preuve en est que son effet le plus durable aura été d'inspirer son Menaud à Félix-Antoine Savard.

Toute œuvre, en nous faisant rêver, nous fait comparer la fausse vie vécue et la vraie vie rêvée dont il s'agit toujours de savoir s'il est obligatoire qu'elle se passe ailleurs, si on ne peut pas la rapatrier ici et aujourd'hui. Comme tout artiste sans doute, mais sur un mode qui lui est propre et qui consiste à vouloir nous familiariser avec l'étrange, l'écrivain venu de loin nous fait rêver, réfléchir. Mais peut-être qu'il nous y force, c'est-à-dire qu'il nous empêche d'éviter de le faire par le dépaysement-repaysement particulier auquel il nous contraint.

\section{Amour lointain}

Qui ne connaît la chanson de Jaufré Rudel intitulée Amour lointain?

Lorsque les jours sont longs en mai j'aime doux chant des oiseaux lointains et, quand de là je suis parti, je me souviens d'un amour lointain...

Je crois à la sincérité du Seigneur. C'est pourquoi je verrai l'amour lointain. Mais, pour un bien qui m'en arrive, j'ai deux maux, tellement il m'est lointain...

J'éprouverai une grande joie en lui dernandant, pour l'amour de Dieu, un gîte lointain et, s'il lui plaît, j'babiterai tout près, moi, le lointain...

Triste et joyeux je laisserai, si je le vois, lamour lointain. Mais je ne sais quand je la reverrai car nos pays sont trop lointains et nombreux sont les passages et les routes... 
Que Dieu qui fit toute créature et forma cet amour lointain, me donne le pouvoir, car mon cour le soubaite, de voir un jour cet amour si lointain...

Il est vrai celui qui m'appelle avide et désireux d'amour lointain, car aucune joie ne me plaît autant que celle d'un amour loin$\operatorname{tain} .$.

C'est peut-être à un tel amour que nous convie l'écrivain qui vient de loin. On ne peut aimer qu'en voyant de loin. Car ce qui se trouve sous nos yeux, comment l'idéaliser? Nous ne pouvons que le regarder simplement. Idéaliser, c'est voir l'idéal sous l'apparence. C'est donc parcourir dans l'objet la distance de la vie à la vraie vie. L'amour est dans le franchissement des apparences, dans le parcours d'une distance, bien souvent par rapport à nousmêmes, dans une distanciation donc, de notre superficialité qui nous attacherait à l'apparence des choses.

Quel peut être cet amour dont le poète dit: "Je me souviens" et dont il précise qu'il est source de deux maux?

Quel sera cet amour qui comblera le poète quand, dans un gite lointain, il habitera tout près, lui qui pourtant demeurera le lointain?

Et pour finir: quel est cet amour dont il est dit qu'il est l'œuvre même de Dieu qui fit toute créature et forma cet amour lointain?

Le passage clé de cette chanson est peut-être celui où il est dit: "je ne sais quand je la reverrai car nos pays sont trop lointains et nombreux sont les passages et les routes". "Il n'est pas d'amour heureux" a cru bon d'expliquer un poète d'aujourd'hui dans sa propre chanson d'amour. De par l'éloignement des pays que nous portons dans nos cours et la multiplicité des routes et passages à emprunter pour les relier, il n'y a pas d'amour aisé, aurait dû préciser ce moderne trop vite désespéré.

Le coeur humain, aux yeux de l'auteur de la chanson médiévale, n'est desireux que d'amour lointain car seul un tel amour le comblerait de joie, lui permettrait de concilier les inconciliables. En attendant il ne peut que se souvenir, avoir la nostalgie, la saudade.

Tout amoureux dit: "je me souviens". Nous tous, en fait, nous le disons et le répétons. Peut-être même que c'est la seule parole que nous puissions prononcer à l'unisson. L'écrivain qui 
vient de loin peut nous aider à mieux reconnaitre notre condition commune d'êtres de souvenir et d'amoureux d'objets lointains car il n'est pas de souvenir qui vaille s'il n'est celui d'un amour dont l'éloignement nous force à rejoindre ce qui est hors de notre portée. 